\title{
Visual Stimulus Design for Strabismus in Preverbal Children in Serpong Area as a Prevention of Amblyopia
}

\author{
Yunisa Fitri Andriani ${ }^{1, *}$ Retno Purwanti Murdaningsih ${ }^{1}$ \\ ${ }^{I}$ Visual Communication Design, Universitas Pembangunan Jaya, South Tangerang, 15414, Indonesia \\ *Corresponding author. Email: yunisa.fitri@upj.ac.id
}

\begin{abstract}
Some newborns experience crossed eyes or misaligned eyeballs in the first few months of life. This happens because the muscles that control the child's eyes are still weak. This misaligned eye condition is known as strabismus. If this condition lasts until the child is 3 months old, it could lead to lazy eye or amblyopia. Amblyopia is more difficult to cure. Preferably, before amblyopia occurs, parents help stimulate the child's eye ability to develop properly. One of the stimuli that can train the child's eye muscles to move in harmony and focus on a visible object is to train it to follow an object. In preverbal children case needs particular stimuli because they can't deliver what they see in a word. The majority of parents who lives in Serpong area until now have not realized the importance of stimulating the child's eye to prevent amblyopia. Through this research, it is hoped that it can invite the Mother and Child Hospital in Serpong to start taking an action in the hospital environment that can stimulate the child's eyes as well as socializing parents to start realizing the importance of stimulating the child's eyes. It is also hoped that the hospital can become a medium for parents to get information about the importance of this visual stimulus.
\end{abstract}

Keywords: child's eye, strabismus, visual, hospital, stimulus

\section{INTRODUCTION}

Strabismus is a vision disorder in which one eye deviates from the line of sight. Amblyopia (more precisely, amblyopia ex anopsia, meaning "decreased vision due to disuse") is a partial deficiency of visual acuity in 1 eye usually a strabismic eye. [1] Strabismus, in Indonesia is known as the squint. Usually strabismus is a genetic component, but someone without this derivative from their family can also have strabismus. Strabismus or crossed eyes can occur at any age, woman or man. The characteristics of strabismus are when the two eyes are not focused on one object, but one eye is on the object while the other eye is on the other direction. Strabismus affects $1 \%$ to $3 \%$ of children. This is seen more frequently in children with a history of prematurity; systemic diseases, such as cerebral palsy; genetic syndrome; and a family history of strabismus. [2] The causes of strabismus in children are many, some of which can be very dangerous and have the potential to be eye or life threatening. Therefore, it is very important that strabismus be identified and managed in the right way and at the right time. Amblyopia is often associated with strabismus, and can be a cause or complication of strabismus, so it is necessary to be recognized and treated early to prevent a permanent decrease in visual acuity. [3] Findings in one study found that children with strabismus had lower functional vision and eye-related quality of life than those who were healthy and visually normal. Parents of children with strabismus also experienced decreased quality of life compared to parents whose controls were visually normal. [4]

During the first 2 months, babies' eyes often don't work together well. The baby's eyes may appear crossed or like looking sideways. In most cases, this is normal and will go away on its own. But if one of your baby's eyes is constantly pointing towards the nose or coming out of the nose, or by 3 months of age, the baby's eyes are not working together to focus and track objects, then this could be serious. Eye or vision problems can delay a baby's development. It is important to spot these problems as early as possible so that parents can get the help they need to grow and learn properly. [5] [15]

Babies are born with a complete visual system, but babies still have to learn to use their eyes because the growth of eye structures takes time. During this growth time, parents must understand how their child's vision should develop so that they can detect early if something is not right. [6] Parents can also provide stimuli to help their child's eye development. Because at this time, teachers and children's vision rehabilitation therapists only play this important role. Even though children often don't complain and make vision problems hidden. Considering this condition, visual acuity should be repeated in children in the environment of their daily lives. [6] 


\subsection{Related Work}

According to Julita 2018 in the Andalas health journal, Preferential looking test is used to assess children's visual acuity. This test is very effective in use for preverbal children or children who cannot speak, namely infants up to 11 months of age. This child's visual acuity is assessed based on the child's response to visual stimuli. The visual stimulus in question is applied to the Teller Acuity Card and Cardiff Acuity Test used in this examination test. [7]

The Teller Acuity Card is 17 grey rectangular cards with black and white stripes on one side. Each of these cards measures $25.5 \mathrm{~cm} \times 55.5 \mathrm{~cm}$. In the middle of the card, there is a $4 \mathrm{~mm}$ diameter hole which functions as a peephole to see the child's eye response. How to use it is by showing this card in front of the child. If the child's eyes move towards the black and white lines, this indicates that the child is able to see the lines. Each card has a different line width, the size of the line width is thinned sequentially. This is intended to check the sharpness of the child's eye, if the child's eye can respond to thin lines, the child's eye acuity is very good. Examination using the Teller Acuity Card can be done monocularly or in one eye by closing one eye to see if one eye is experiencing interference. This examination can also be done binoculars or 2 eyes. The distance between the card and the child's eye is determined based on the child's age. Examination for infants aged 0-6 months, the distance between the card and the child's eye was $38 \mathrm{~cm}$, while the examination for children aged 7 months to 3 years, the distance between the card and the child's eye was $55 \mathrm{~cm}$. [8]

Slightly different from the Teller Acuity Card (TAC), the Cardiff Acuity Test (CAT) is a card that contains images that are familiar to children, for example objects they have often seen such as houses, ducks or fish. What it has in common with TAC is the colors used, namely black, white and gray. Objects on the CAT are made with only white outlines and this outline also has a thin black outline. The background of this card is the same as the TAC which is gray. The black outline is half the width of the white outline. The size of each CAT is smaller than the TAC, which is 21 $x 29.5 \mathrm{~cm}$. CAT also does not have a peephole like the TAC and the position of the image is randomly placed on the top or bottom side. [7]

\subsection{Our Contribution}

This research presents the outcome of the development of the preferential looking test design that already exists and has been used until present. [7] This design combines the theory of preferential looking tests with the theory of child development by Prince in his 2020 book that stimuli to the eyes of children must be given daily in the environment and involve the people around them. The design of the visual stimulus will be adapted to the case in a mother and child hospital in the serpong area. [6]

\subsection{Paper Structure}

Other papers are organized as follows. Section 2 is a description of several theories that underlie the design, namely the theory of strabismus and amblyopia, the results of the survey to parents in the serpong area and the results of interviews with ophthalmologists at the Serpong Mother and Child Hospital. Section 3 present the stimulus visual design for preverbal children that applied in Children and Women Hospital in Serpong area.

\section{STRABISMUS AND AMBLYOPIA}

Strabismus, more commonly known as crossed eyes, occurs when there is a misalignment of the eyes. [3] Strabismus, which is most often a developmental disorder, is characterized by abnormal eye alignment. At all times, the strabismic subject used one eye to gaze and acquire information while the other eye (diverge) was not fixated. Strabismic subjects with amblyopia (reduced visual acuity) prefer to use their non-amblyopic eye to fixate on the target. [9] Strabismus affects $1 \%$ to $3 \%$ of children. It is seen more frequently in children with a history of prematurity, systemic diseases, such as cerebral palsy, genetic syndromes, and a family history of strabismus. [10] Physical assessment and eye acuity in children were carried out based on age. In infants this is done with a simple torch, observe the corneal reflex while moving it from side to side. The middle corneal light reflex indicates "central" fixation, and the continuation of the eye to the side of the torch is referred to as "stable", and if the eyes are parallel during movement, this is referred to as "maintained". Whereas for preverbal children, preferential performance tests were carried out, for example, the Cardiff card. The prognosis for strabismus is excellent if detected and treated early. At about three months, normal binocular coordination occurs, and any strabismus that persists after this period should be considered abnormal and significant. Amblyopia occurs when strabismus is not treated properly before 6-8 years which causes permanent deterioration of vision. Parents should know the prognosis, benefits, and complications of strabismus management and its impact on the child's social, physical and psychological growth. A care plan based on discussion with parents should be mapped. Parents need to be aware of the risk of developing amblyopia and stereopsis disorder if medication adherence is not maintained. [11] [14]

As has been mentioned above, the treatment for children's eye acuity should be carried out in an everyday environment. The strabismus book also states that the treatment of strabismus in children involves a multidisciplinary approach and may occur in various locations. The groups involved are patients and family members, hospital administrators, medical personnel, orthopedists and ophthalmologists, community pediatricians, general practitioners, and health visitors. Apart from these varied groups, teachers, school nurses and community ophthalmologists also play an important role in its management. [11] [13]

Amblyopia is a visual development disorder consisting of decreased visual acuity in one eye or rarely in both eyes in the absence of ocular pathology. Due to the abnormalities in visual processing that occur in amblyopia, there are also deficits in contrast sensitivity, accommodation, binocular vision, fixation, saccades, colour, and shape and motion 
perception. [12] People suffering from strabismus struggle with visual spatial orientation. This orientation is a mental phenomenon present in the visual cortex in the brain. If left untreated, this strabismus eye will experience a decrease in visual acuity, a condition known as amblyopia or lazy eye. [6]

Conventional treatments for amblyopia are goggles, patches, and punishments with atropine drops or Bangerter filters, but in recent years new approaches based on computerized visual stimuli using different types of stimuli have been developed and evaluated. [12] [14]

\section{PARENTAL SURVEY}

Parents can also provide stimuli to help their child's eye development. Because parents should take an important role in stimulating the child's eyes, this study conducted a survey of parents. The survey is distributed to parents who live in Serpong and its surroundings. The results of the survey are used as a basis for the information that will be included in the visual. The questions and answers from the survey are explained in the diagram below:

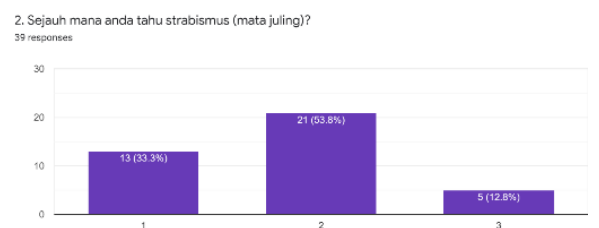

Diagram 1 Survey How Much Parents Know About Strabismus

The majority of parents in Serpong and surrounding areas do not know what strabismus is. Looking at the middle diagram, it can be said that the Parents know Strabismus but do not understand it. The next question in this survey is about the age at which strabismus usually occurs.

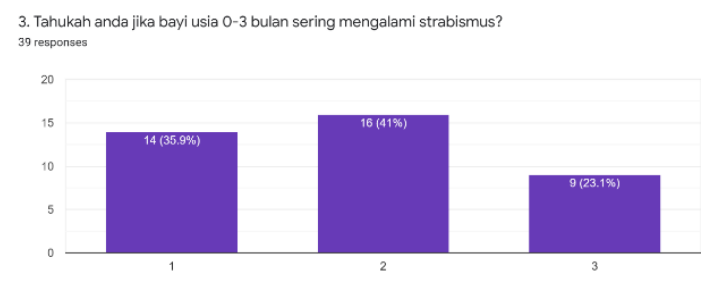

Diagram 2 Survey of Parents' Knowledge About the Common Age of Strabismus

Just like the previous question, the majority of parents did not know that at 0-3 months of age children often experience strabismus. The next question is about amblyopia.

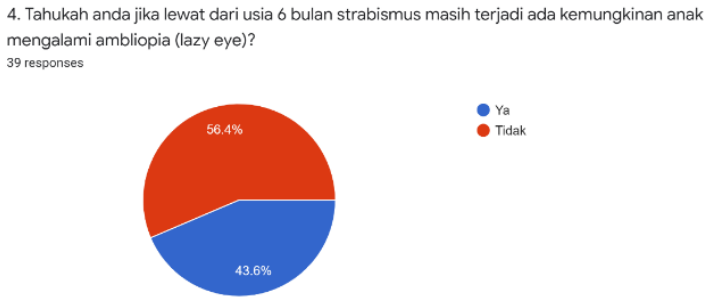

Diagram 3 Survey How Much Parents Know Strabismus Can Cause Amblyopia

Most parents do not know that amblyopia can occur if strabismus is not treated. Next, the parents were asked questions about the stimulus that should be given to the child to prevent amblyopia from occurring.

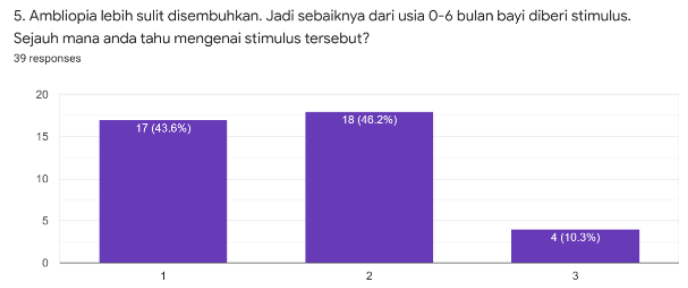

Diagram 4 Survey The extent of parents' knowledge about children's eye stimuli

From the survey that has been carried out, it can be said that the parents' knowledge about strabismus, amblyopia and stimuli is still lacking. Meanwhile, the dominant environment for children is their environment with their parents. So, we need a media that provides information to parents about the stimulus needed for children's eye development.

\section{OPHTHALMOLOGIST INTERVIEW}

This interview was conducted via telephone due to the pandemic conditions which made it impossible for inperson interviews. The following is an explanation of the results of an interview with an ophthalmologist at Hermina Serpong Hospital. The number of cases of visual impairment in children at Hermina Serpong Hospital is less than 5 cases per month. Usually the most cases are refractive errors. The majority of visual disturbances in children occur due to genetic factors. According to ophthalmologists, newborns cannot see clearly so that it is still difficult to provide visual stimuli. At the age of 6 months, the child has begun to see clearly and can be given stimuli to detect whether the child's eyes are sharp or not. The usual stimulus is an attractive picture pasted on the wall of the doctor's office. If the child's eyes do not follow the object shown, further examination is necessary. The doctor also explained that the image shown should attract the child's eye, which is a simple shape, brightly coloured like red, and large in size. The researcher also asked how to use the mural to apply these stimuli. The doctor explained that this could be done considering the large wall so that it is 
suitable for applying objects that attract the child's eye, besides that there are not many hospitals that have implemented this.

\section{Visual Stimulus Design}

The following are some sketches of visual stimulus designs that have been made in accordance with the results of interviews and the preferential looking test method. Furthermore, it still requires validation from the ophthalmologist whether this visual stimulus design is good and appropriate.

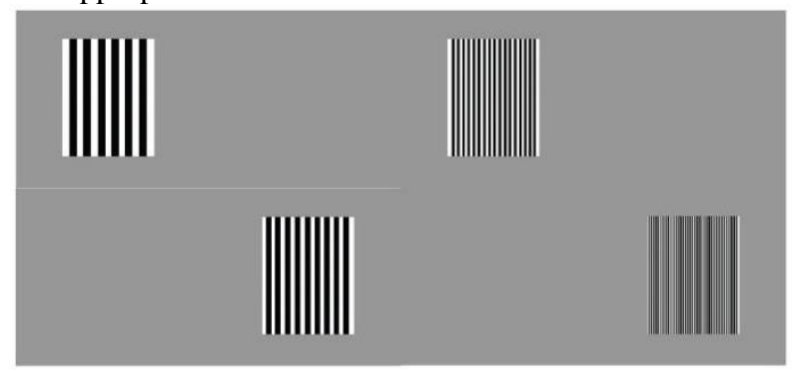

Figure 3.1 Design 1

Design 1 is made with the visual concept of the Teller Acuity Card method. The colours used are also the same as the colours on the TAC. The shape concept is made accordingly, with only black and white lines on one side. The difference between this design and the original TAC was the application and the absence of a peephole in the center. TAC is designed to measure children's eye acuity. While this design is made as a child's eye stimulus so that it does not require a peephole in the middle, and the arrangement of the card into a patterns that decorate the hospital walls.

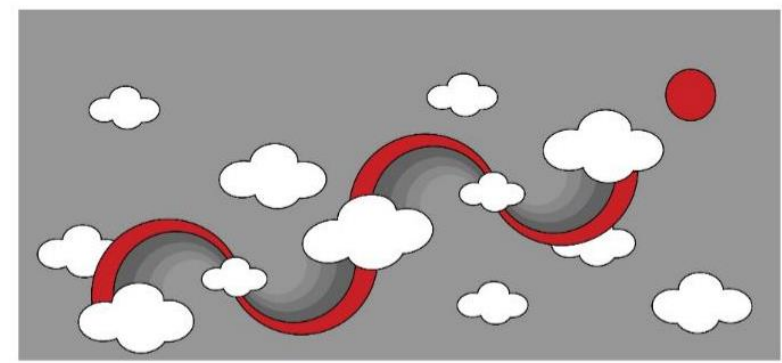

Figure 3.2 Design 2

Design 2 combines the visual concept of TAC, CAT, and the results of the ophthalmologist's interview that children aged 6 months and over prefer bright colours and attractive shapes. However, the shapes here are made with a simple pattern and still a collection of lines. It is hoped that with this shape the child's eyes can follow the direction of the rainbow line.

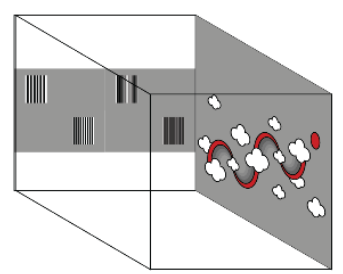

Figure 3.3 Application of the Design to the Hospital Wall The use of murals as a visual stimulus medium for children's eyes was chosen based on the results of discussions in interviews with ophthalmologists. Considering a large wall size according to the visual size that attracts the child's eye. Murals can also be used as a medium of information for parents about how important it is to stimulate children's eyes as well as information about strabismus and amblyopia that can occur in children's eyes

\section{CONCLUSION}

The methods used in this study included interviews of ophthalmologists, surveys of parents and literature studies. Through the interview method, it can be concluded that hospitals in Indonesia, especially in the serpong and surrounding areas, have not utilized the hospital walls as a medium that contains visual stimuli as well as information for parents. Meanwhile, through the survey method, it can be concluded that parents, especially in the serpong and surrounding areas, do not understand the importance of stimuli in preventing amblyopia. Through literature studies, the conclusion that can be drawn is that the development of preverbal children requires a special stimulus to detect eye acuity. The Preferential Looking Test is currently the most effective method for use in preverbal children.

\section{ACKNOWLEDGMENT}

This research is supported and financed by Research and Community Service Unit, Universitas Pembangunan Jaya, Indonesia.

\section{REFERENCES}

[1] McLaughlin, S. C. (2016). Visual perception in strabismus and amblyopia. APA PsycArticles.

[2] Gunton, K. W. (2015). Strabismus. Primary Care. PubMed.gov.

[3] Tejaswi Bommireddy, K. T. (2020). Assessing strabismus in. Paediatrics and Child Health, 14-18 [4] Sarah R. Hatt, D. D. (2020). Association of Strabismus With Functional Vision and Eye-Related Quality of Life in Children. JAMA Ophthalmology. [5] Boyd, K. (2020, July 8). Vision Development: Newborn to 12 Months. Retrieved from www.aao.org: https://www.aao.org/eye-health/tips-prevention/babyvision-development-first-year

[6] Prince, L. G. (2020). Manual for Child and Vision Development Milestone. Xlibris. 
[7] Julita. (2018). Pemeriksaan Tajam Penglihatan pada Anak dan Refraksi Siklopegik: Apa, Kenapa, Siapa?

Jurnal Kesehatan Andalas.

[8] R. T. Mackie', K. J. (1996). Visual acuity

assessment of children with neurological impairment

using. ACTA OPHTHALMOLOGICA

SCANDINAVICA.

[9] Ramachandran, S., \& Das, V. E. (2020). Fixation

Preference for Visual and Auditory Targets in Monkeys with Strabismus. iovs.

[10] Gunton, K. W. (2015). Strabismus. Primary Care.

PubMed.gov.

[11] Kanukollu, V. M., \& Sood., G. (2020). Strabismus. StatPearls Publishing LLC.

[12] Carlos J. Hernández-Rodríguez, D. P. (2020).

Active Vision Therapy for Anisometropic Amblyopia

in Children: A Systematic Review. Journal of

Ophthalmology.

[13] Nicola Herbison, D. M. (2016). Randomised controlled trial of video clips and interactive games to improve vision in children with amblyopia using the IBiT system. PubMed.

[14] Oren Yehezkel, M. B.-J. (2020). Automated Diagnosis and Measurement of Strabismus in Children. American Journal of Ophthalmology.

[15] Soedjatmiko. (2006). Pentingnya Stimulasi Dini untuk Merangsang Perkembangan Bayi dan Balita Terutama pada Bayi Risiko Tinggi. Sari Pediatri, Vol.8, No.3. 